\title{
Simulation of Waves Processes in Dusty Emission of Volcano
}

\author{
V.V. Grimalsky ${ }^{1}$, M.A. Cruz Chavez ${ }^{1}$, S.V. Koshevaya ${ }^{1}$, A.Kotsarenko ${ }^{2}$, \\ M.Hayakawa ${ }^{3}$, R. Pérez Enriquez ${ }^{2}$ \\ ${ }^{1}$ Autonomous University of Morelos (UAEM), Cuernavaca ZP 62209, Mor., Mexico \\ ${ }^{2}$ Center of Geoscience, UNAM, Juriquilla, Qro., ZP 76230, Mexico \\ ${ }^{3}$ The University of Electro-Communication, Chofu, Tokyo 182, Japan \\ E-mail:leniem@gmail.com,tgkim@chonnam.ac.kr \\ Received February 4, 2011; revised March 8, 2011; accepted March 29, 2011
}

\begin{abstract}
A general method of simulation of processes in dusty based on special programs is presented here. It is possible to prepare the modeling of the dusty in volcano like the dust sound waveguides. Dusty is in state of the plasma. Waveguides are formed by the distribution of dusty particles with various masses $m=m(x)$ in transverse coordinate. The dust sound waves propagate along the longitudinal $z$-direction. In the case of contact of dusty plasma with a semi-infinite dielectric, there exists the dust acoustic mode that possesses the negative group velocity (backward wave) in the specified interval of wave numbers. For analysis it is necessary to use the special numerical methods of calculation of the equations with boundary conditions. Simulation of ion sound wave propagation shows a new dispersion between frequency and wave vector. In some region of parameters of dusty the negative dispersion of wave takes place. This means that the phase and group velocities of wave are opposite (negative dispersion). This phenomenon takes place, when the mass of dust particles has the maximum in the center of the waveguide. The negative dispersion caused the instability in dusty, which open the possibility to create a new phenomenon in dusty including the high temperature and the flame.
\end{abstract}

Keywords: Dusty Plasma, Dispersion, Negative Group Velocity

\section{Introduction}

Nowadays, dusty, both in space and under laboratory conditions are the object of numerous investigations [1-6]. Besides traditional investigation of dusty plasmas in connected with it influence to communication, flights of airplanes and people living near volcano and another eruptions. There are also the artificial dust clouds in the atmosphere caused by industrial pollutions, very danger for communication and for flights. An attractive property of dusty plasmas is supporting various oscillations and waves, both linear and nonlinear [2,3,7-11]. There are a lot of papers devoted to the waves in bounded or inhomogeneous plasmas, where some types of surface plasma waves can exist and the presence of the dust component can affect essentially their characteristics [5-12]. A distinctive property of the dust component is a possibility to get plasmas with dust particles of various properties in different parts of the system [1-3,5]. For instance, it is possible to consider a situation when along a certain direction the mass of the particles varies. The dependence of the mass of the particle on the coordinate should influence on the properties of the waves propagating perpendicularly to such an axis.

This paper is devoted to simulation of wave's processes of surface dust acoustic waves in the dusty plasma with the dependence of the mass of dust particles on the transverse coordinate. It is found that the variable mass of the particles affects the properties of the linear surface dust acoustic waves. Namely, when the heavier particles are near the interface, it is possible to observe the anomalous wave dispersion (backward waves). Additionally, in this case the peculiarities of the dependence of transverse electric field on the transverse coordinate $x$, like non-monotonic transverse profile, can occur. Also the variable masses lead to a mechanism of nonlinearity under propagation of surface waves of finite amplitudes. For simulation of wave processes it is created the com- 
puting method which take into account the boundary conditions in no homogenous dusty.

\section{Model of Dusty Plasma}

Consider the dusty plasma that includes positive ions and negative dust. The temperature of ions is finite whereas the temperature of chaotic motion of the dust is lower. Note that the temperature of the dust particles, which are much more massive that the ions, is the characteristic of their chaotic motion, it is not the temperature within a single dust particle.

The dust acoustic waves in the bounded plasma can be described by hydrodynamic equations for ions and dust added by boundary conditions. Below, we consider the waves that propagate along the interface between the dusty plasma $(x>0)$ and the dielectric with a permittivity $\varepsilon(x<0)$, see Figure 1 .

Generally, the particles of the dust in different parts of the plasma are different and may have different masses. First of all it is necessary to formulate the basic equations and boundary conditions for an analysis of the dusty plasma waveguide with the particles of variable masses. It is necessary to consider the dusty plasma with the following components: negative dust $\left(m_{d},-Q\right)$ and positive ions $\left(m_{i}, e\right)$. The charge of each dust particle is $-Q=-Z e, Z \gg 1$. The temperatures of the components are $T_{d} \ll T_{i} \neq 0$. The practical interest has the case when the particles of the dust have different masses: $m_{d}=$ $m_{d}(x)$. Here $x$ is the initial $x$-coordinate of the particle. Real model of dust is the case no homogeneous plasma of the following masses dependence:

$$
m_{d}(x)=m_{d \infty} \cdot\left[1+a_{0} \times \exp \left(-\left(\frac{x}{x_{0}}\right)^{2 p}\right)\right]
$$

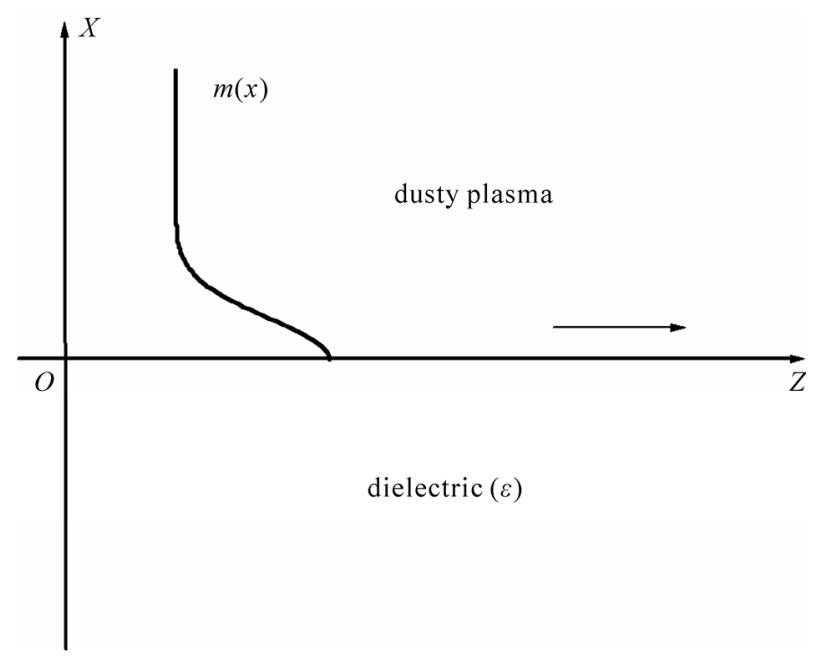

Figure 1. Geometry of the problem. where the constant $a_{0}$ has the values within the interval $-1<a_{0}<10$. In the homogeneous plasma it is the case that the constant $a_{0}=0$. The parameter $m_{d \infty}$ is the mass of the particle far from the interface $x=0$. The parameter $p$ is $p=1$ or 2 (case 1 is the Gaussian profile; case 2 is supergaussian one, or step-like).

The propagation of dust acoustic surface waves is investigated, when this propagation is along $z$-axis takes place, whereas in $x$-direction the localization occurs.

The hydrodynamic equations for the dust component are $[2,3]$ :

$$
\begin{aligned}
& \frac{\partial n_{d}}{\partial t}+\operatorname{div}\left(n \boldsymbol{V}_{d}\right)=0 \\
& \frac{\mathrm{d} \boldsymbol{V}_{d}}{\mathrm{~d} t} \equiv \frac{\partial \boldsymbol{V}_{d}}{\partial t}+\left(\boldsymbol{V}_{d} \cdot \nabla\right) \boldsymbol{V}_{d}=-\frac{Q}{m_{d}} \boldsymbol{E}-\frac{k_{B} T_{d}}{m_{d} n_{d}} \nabla n_{d} \\
& \frac{\mathrm{d} u_{x}}{\mathrm{~d} t}=V_{d x} ; m_{d} \equiv m_{d}\left(x-u_{x}\right)
\end{aligned}
$$

One can see that in the hydrodynamic equations for the dust there is an additional source of nonlinearity, due to the dependence of the mass of the particle on the displaced transverse coordinate $x$.

For the electric field, the Poisson equation takes the form:

$$
\Delta \varphi=4 \pi \cdot\left(-e n_{i}+Q n_{d}\right) ; \boldsymbol{E}=-\nabla \varphi .
$$

The condition of quasi-neutrality of the charge is $-e n_{i 0}+Q n_{d 0}=0$.

For ions, also we use the hydrodynamic equations:

$$
\frac{\partial n_{i}}{\mathrm{~d} t}+\operatorname{div}\left(n_{i} \boldsymbol{V}_{i}\right)=0 ; m_{i} n_{i} \frac{\mathrm{d} \boldsymbol{V}_{i}}{\mathrm{~d} t}=-e n_{i} \nabla \varphi-k_{B} T_{i} \nabla n_{i}
$$

But, because of the inequality $m_{i} \ll m_{d}$, it is possible to consider the validity of the Boltzmann distribution for ions:

$$
n_{i}=n_{i 0} \exp \left(-\frac{\varphi}{\varphi_{T i}}\right) ; \varphi_{T_{i}}=\frac{k_{B} T_{i}}{e}
$$

Therefore, the Poisson equation can be simplified:

$$
\Delta \varphi=4 \pi\left(-e \cdot n_{i 0} \exp \left(-\frac{\varphi}{\varphi_{T i}}\right)+Q n_{d}\right)
$$

The waves of small amplitudes are considered; therefore, the linearized equations are utilized below. Note that in the linealized equations it is necessary to use the dependence of $m_{d}$ on $x$ only.

\section{Basic Equations}

The derivatives in Equations (3) and in the boundary conditions (1)-(6) have been approximated by the undimensional linearized equations (the index $d$ near $V, m$, 
and $n$ is omitted) can be represented as:

$$
\begin{aligned}
& \frac{\partial \boldsymbol{V}}{\partial t}=\frac{1}{m(x)} \nabla \varphi-\frac{T}{m(x)} \nabla \tilde{n} ; \Delta \varphi=\varphi+\tilde{n} ; \\
& \frac{\partial \tilde{n}}{\partial t}+\operatorname{div} \boldsymbol{V}=0 ; \tilde{n} \equiv n-n_{0}
\end{aligned}
$$

The following nondimensional variables have been applied: $x \rightarrow x / r_{D i}, z \rightarrow z / r_{D i}, V \rightarrow V / V_{n}, t \rightarrow t / t_{n}, \varphi \rightarrow$ $\varphi / \varphi_{T i}, m \rightarrow m / m_{\infty}, \tilde{n} \rightarrow \tilde{n} / n_{d 0}, T=T_{d} e / T_{i} Q \ll 1$. Here the scale of the distance $r_{D i}=\left(k_{B} T_{i} / 4 \pi e^{2} n_{i 0}\right)^{1 / 2}$ is the Debye radius for ions, the characteristic velocity is $V_{n}=$ $\left(Q k_{B} T_{i} / e m_{\infty}\right)^{1 / 2}$, the time scale is $t_{n}=r_{D i} / V_{n}$. Therefore, the undimensional mass of the dust particle is $m(x)=$ $1+a_{0} \exp \left(-\left(x / x_{0}\right)^{2 p}\right)$. Note that the undimensional dust temperature is a product of two small magnitudes $T_{d} / T_{i}<$ 1 and $e / Q \sim 10^{-3} \cdots 10^{-6}$, therefore, $T<10^{-3}$.

It is necessary to use the following algorithm of solution. First of all the traveling wave has view $\varphi, \tilde{n} \sim$ $\exp (\mathrm{i}(\omega t-k z))$.In this case, the set of equations (7) can be reduced to the equations for the potential and for variable part of the dust concentration:

$$
\begin{aligned}
& \frac{\mathrm{d}}{\mathrm{d} x}\left(\frac{1}{m(x)} \frac{\mathrm{d} \tilde{n}}{\mathrm{~d} x}\right)-\left(\frac{k^{2}}{m(x)}-\frac{\omega^{2}}{T}\right) \tilde{n} \\
& -\frac{1}{T} \frac{\mathrm{d}}{\mathrm{d} x}\left(\frac{1}{m(x)} \frac{\mathrm{d} \varphi}{\mathrm{d} x}\right)+\frac{k^{2}}{m(x) T} \varphi=0 \\
& \frac{\mathrm{d}^{2} \varphi}{\mathrm{d} x^{2}}-\left(1+k^{2}\right) \varphi-\tilde{n}=0 .
\end{aligned}
$$

It is necessary to add Equations (8) by the boundary conditions. The continuity of the electric potential, the $x$-component of the electric field (absence of the surface charge at $T \neq 0$ ), and zeroing $v_{x}$-component of dust velocity result in the boundary conditions at $x=+0$ :

$$
\frac{\mathrm{d} \varphi}{\mathrm{d} x}-\varepsilon k \varphi=0 ; \frac{\mathrm{d} \varphi}{\mathrm{d} x}-T \frac{\mathrm{d} \tilde{n}}{\mathrm{~d} x}=0 .
$$

Another boundary conditions are at $x=L_{x} \gg x_{0}$. In the region $x \ll x_{0}$ the value of the nondimensional dust mass is $m_{\infty}=1$. In this region it is possible to reduce Equations (8):

$$
\begin{aligned}
& \frac{\mathrm{d}^{2} \tilde{n}}{\mathrm{~d} x^{2}}-\left(k^{2}+\frac{1-\omega^{2}}{T}\right) \tilde{n}-\frac{1}{T} \varphi=0 \\
& \frac{\mathrm{d}^{2} \varphi}{\mathrm{d} x^{2}}-\left(1+k^{2}\right) \varphi-\tilde{n}=0 \\
& \left.\frac{\mathrm{d}^{2} \varphi}{\mathrm{d} x^{2}}\right|_{x=x_{j}} \approx \frac{1}{h^{2}}\left(\varphi_{j+1}-2 \varphi_{j}+\varphi_{j-1}\right) ; \\
& \left.\frac{\mathrm{d}}{\mathrm{d} x}\left(\frac{1}{m(x)} \frac{\mathrm{d} \tilde{n}}{\mathrm{~d} x}\right)\right|_{x=x_{j}} \approx \frac{1}{h^{2}}\left(\frac{1}{m\left(x_{j+1 / 2}\right)}\left(\tilde{n}_{j+1}-\tilde{n}_{j}\right)-\frac{1}{m\left(x_{j-1 / 2}\right)}\left(\tilde{n}_{j}-\tilde{n}_{j-1}\right)\right) ; \\
& \left.\frac{\mathrm{d} \varphi}{\mathrm{d} x}\right|_{x=x_{N}} \approx \frac{1}{h}\left(\varphi_{N}-\varphi_{N-1}\right), \text { where } \varphi_{j} \equiv \varphi\left(x_{j}\right), x_{j} \equiv j \cdot h
\end{aligned}
$$

\section{Simulation Method}

The solution of (10) has the character to decreases at $x \gg x_{0}$ like $\varphi, \tilde{n} \sim \exp (-\tau x), \tau>0$. It takes the form:

$$
\begin{aligned}
& \varphi=A_{1} \exp \left(-\tau_{1} x\right)+A_{2} \exp \left(-\tau_{2} x\right) \\
& \tilde{n}=\alpha_{1} A_{1} \exp \left(-\tau_{1} x\right)+\alpha_{2} A_{2} \exp \left(-\tau_{2} x\right) \\
& \text { where } \alpha_{1,2}=\tau_{1,2}^{2}-k^{2}-1
\end{aligned}
$$

In case of $\tau_{1,2}>0$ the solutions have following view:

$$
\left(\tau^{2}-k^{2}\right)^{2}+\left(\tau^{2}-k^{2}\right)\left[\frac{1}{T}\left(\omega^{2}-1\right)-1\right]-\frac{\omega^{2}}{T}=0 .
$$

Excluding the constants $A_{1,2}$ from (11), one can get the following boundary conditions at $x=L_{x} \gg x_{0}$ :

$$
\begin{aligned}
& \frac{\mathrm{d} \varphi}{\mathrm{d} x}+\frac{\tau_{2} \alpha_{1}-\tau_{1} \alpha_{2}}{\alpha_{1}-\alpha_{2}} \varphi+\frac{\tau_{1}-\tau_{2}}{\alpha_{1}-\alpha_{2}} \tilde{n}=0 ; \\
& \frac{\mathrm{d} \tilde{n}}{\mathrm{~d} x}+\frac{\alpha_{1} \alpha_{2}\left(\tau_{2}-\tau_{1}\right)}{\alpha_{1}-\alpha_{2}} \varphi+\frac{\alpha_{1} \tau_{1}-\alpha_{2} \tau_{2}}{\alpha_{1}-\alpha_{2}} \tilde{n}=0 .
\end{aligned}
$$

It is necessary begin with simplified model where parameter $T=0$. It is possible to obtain the single equation for the potential only there:

$$
\frac{\mathrm{d}}{\mathrm{d} x}\left[\varepsilon_{d}(x) \frac{\mathrm{d} \varphi}{\mathrm{d} x}\right]-\left(k^{2} \varepsilon_{d}(x)+1\right) \varphi=0 .
$$

A notation is used for the effective permittivity of the plasma due to the dust component: $\varepsilon_{d}(x)=1-1 /\left(m(x) \omega^{2}\right)$. To get the waves localized in $x$-direction, it is necessary to satisfy the condition $\varepsilon_{d}(x)<0$.

The boundary condition at $x=+0$ is:

$$
\varepsilon_{d}(x=0) \frac{\mathrm{d} \varphi}{\mathrm{d} x}-\varepsilon k \varphi=0,
$$

because the potential and $x$-component of the electric induction are continuous there.

At $x=L_{x} \gg x_{0}$, it is possible to get the boundary condition as:

$$
\frac{\mathrm{d} \varphi}{\mathrm{d} x}+\tau \varphi=0, \tau^{2}=\frac{1}{\varepsilon_{d \infty}}+k^{2}>0, \varepsilon_{d \infty} \equiv 1-\frac{1}{\omega^{2}} .
$$

For the numerical solution of Equation (8) added by boundary conditions (9) and (12), we have used the finite 
Here $h$ is the step along $O X$ axis; $L_{x}=N \cdot h$.

As a result, a set of uniform linear equations has been formed. They possess a matrix structure:

$$
\begin{aligned}
& \hat{\alpha}_{-1, j} \boldsymbol{A}_{j-1}+\hat{\alpha}_{0, j} \boldsymbol{A}_{j}+\hat{\alpha}_{+1, j} \boldsymbol{A}_{j+1}=0, \\
& j=1, \cdots, N-1 ; \\
& \hat{\alpha}_{0,0} \boldsymbol{A}_{0}+\widehat{\alpha}_{0,+1} \boldsymbol{A}_{1}=0, \\
& \hat{\alpha}_{-1, N} \boldsymbol{A}_{N-1}+\hat{\alpha}_{0, N} \boldsymbol{A}_{N}=0 ; \boldsymbol{A} \equiv\left(\begin{array}{c}
\varphi \\
\tilde{n}
\end{array}\right)
\end{aligned}
$$

Here the coeficients $\hat{\alpha}_{-1, j}$ etc. are matrices $2 \times 2$. This set of equations has been solved by the factorization method. Namely, it is possible to represent:

$$
\boldsymbol{A}_{j}=\hat{\beta}_{j} \boldsymbol{A}_{j-1}, \quad j=N, \cdots, 1 ;
$$

and to calculate the matrices $\hat{\beta}_{j}$ consecutively, step by step. As a result, at $j=0$ it is possible to get a single matrix equation for $\boldsymbol{A}_{0}$ as:

$$
\left(\widehat{\alpha}_{0,0}+\widehat{\alpha}_{+1,0} \hat{\beta}_{1}\right) A_{0}=0
$$

It is necessary to search for nontrivial solutions: $\boldsymbol{A}_{0} \neq 0$. Therefore, the following determinant is equal to zero:

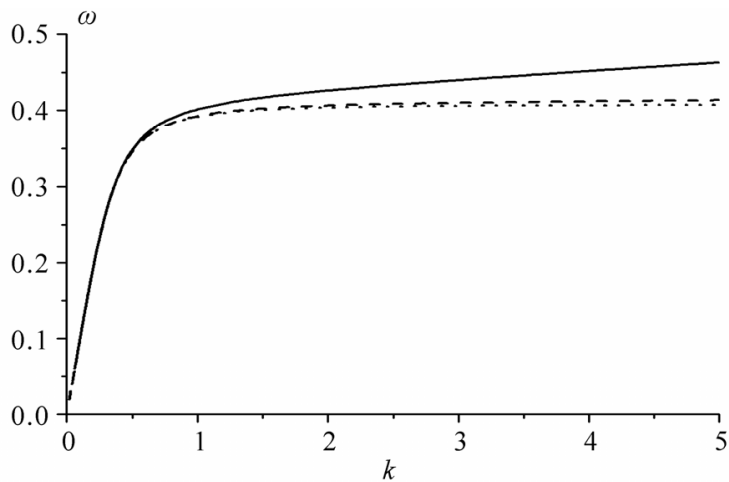

(a)

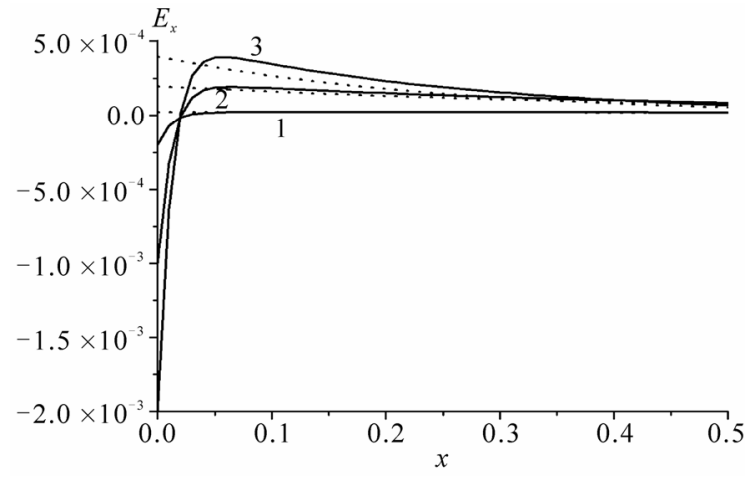

(c)

$$
\operatorname{det}\left(\left(\hat{\alpha}_{0,0}+\hat{\alpha}_{+1,0} \hat{\beta}_{1}\right)\right)=0
$$

This is the dispersion equation for the set of dispersion curves $\omega=\omega_{m}(k), m=1,2, \cdots$ The interst of our modeling is to obtain the lowest (fundamental) mode, which can be observed in experiments.

For the simplified version, Equations (13), (14), (15), the method is the same, but the matrices are reduced to scalars.

\section{Results of Simulation and Discussing}

Using Equation (8) and boundary conditions (9), (12), it is possible to simulate the dispersion curves and the transverse profiles of the surface dust acoustic waves. The shooting method has been applied to solve the ordinary differential equations jointly with boundary conditions. The main attention has been paid to the qualitative effects of the variable masses of dust particles on the propagation of the surface dust waves. Below, the results of simulations are presented for different parameters of the dust. The results are given for the undimensional magnitudes in Figures 2-5. The simulations have been

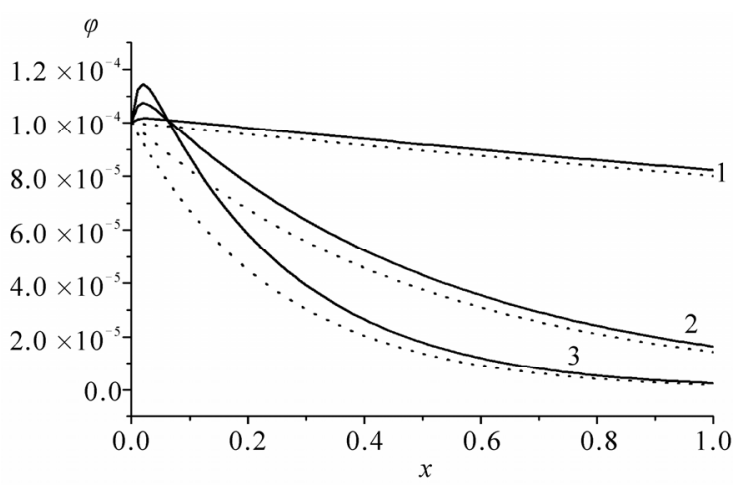

(b)

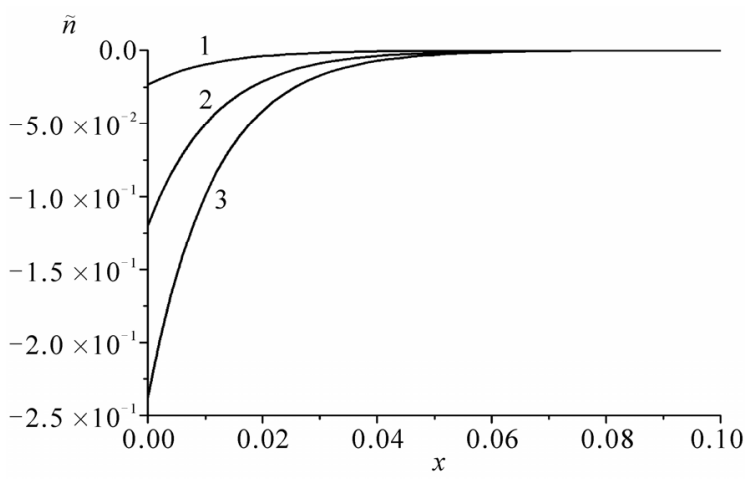

(d)

Figure 2. The dispersion curve $\omega(k)(a)$, transverse profiles of the potential $\varphi(b), x$-component of the electric field $E_{x}$ (c), variable part of concentration $\tilde{n}\left(\right.$ for $\left.T=10^{-4}\right)(\mathrm{d})$. Solid curves are for $T=10^{-4}$, dash curves are for $T=10^{-6}$, dot curves are for $T$ $=0$. The parameters are as follows: $a_{0}=0$ (uniform plasma at $x>0$ ), $\varepsilon=5$. The curves $1,2,3$ correspond to the values of the wave numbers $k=0.4, k=2, k=4$. 


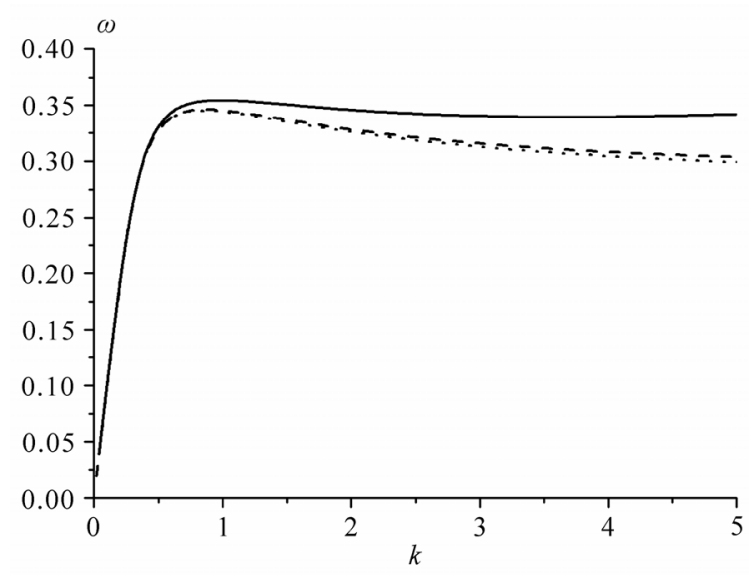

(a)

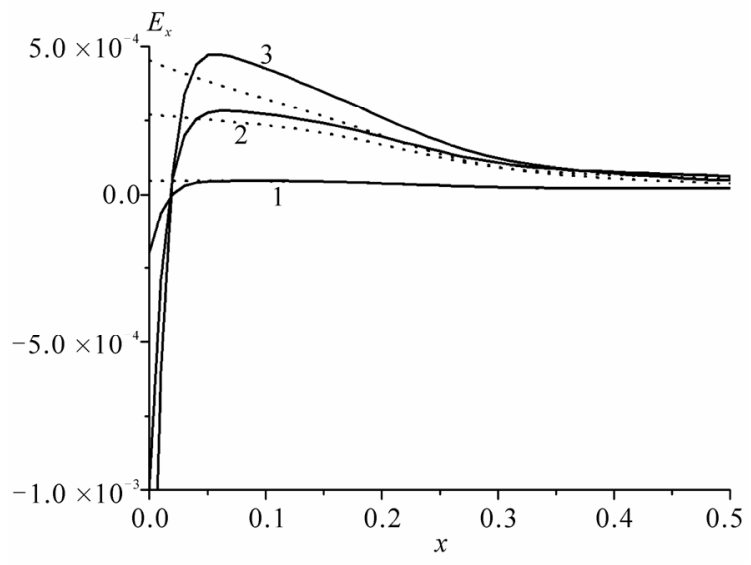

(c)

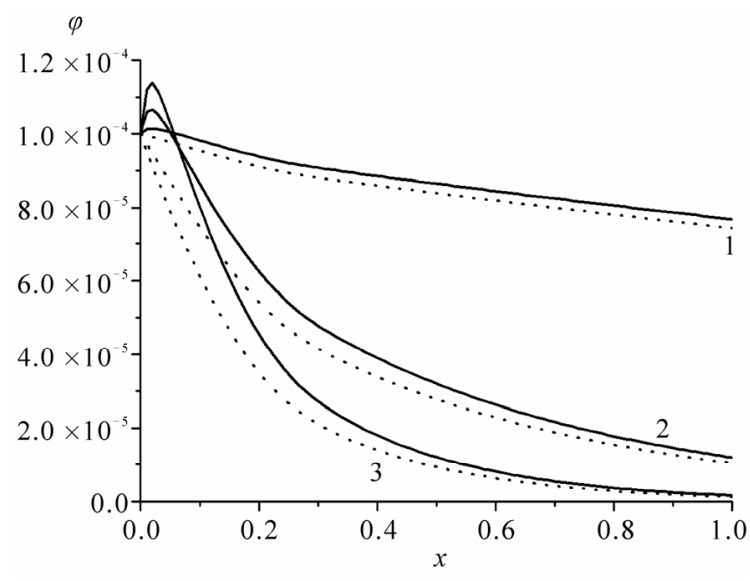

(b)

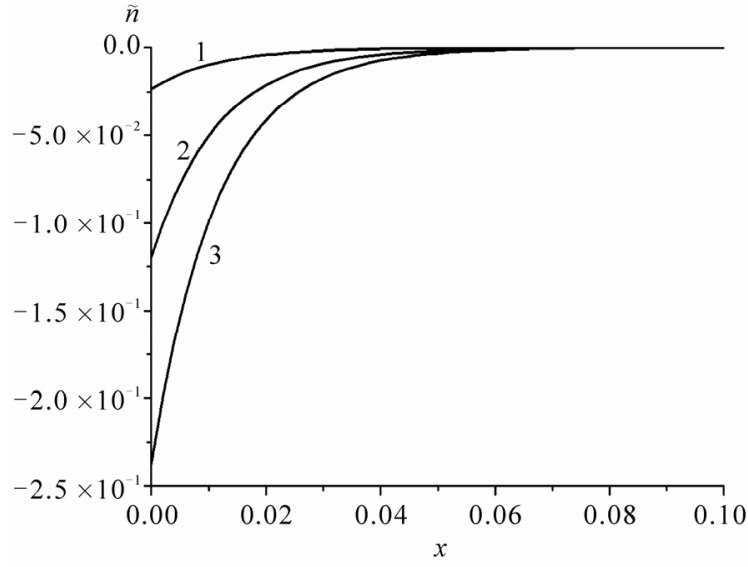

(d)

Figure 3. The dispersion curve $\omega(k)(a)$, transverse profiles of the potential $\varphi(b), x$-component of the electric field $E_{x}(c)$, variable part of the dust concentration $\tilde{n}\left(\right.$ for $\left.T=10^{-4}\right)(\mathrm{d})$. Solid curves are for $T=10^{-4}$, dash curves are for $T=10^{-6}$, dot curves are for $T=0$. The parameters are as follows: $a_{0}=1$ (heavier particles in the center of the waveguide), $\varepsilon=5, x_{0}=0.25, p=2$. The curves $1,2,3$ correspond to the values of the wave numbers $k=0.4, k=2, k=4$.
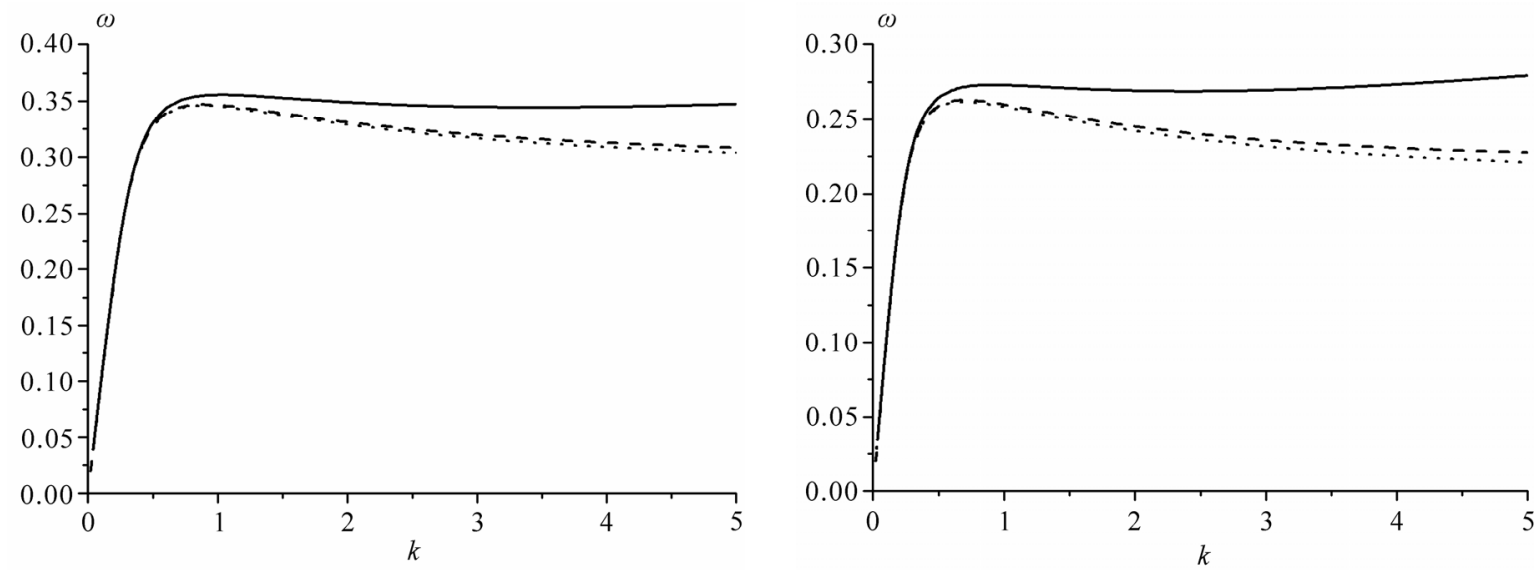

(a) (b)

Figure 4. The dispersion curves $\omega(k)$ for dusty plasma waveguides with the heavier particles in the center of the waveguide. Solid curves are for $T=10^{-4}$, dash curves are for $T=10^{-6}$, dot curves are for $T=0$. The parameters are: (a) $a_{0}=1, \varepsilon=5, x_{0}=$ $0.25, p=1$ (Gaussian profile); (b) $a_{0}=1, \varepsilon=10, x_{0}=0.25, p=2$ (step-like profile). 


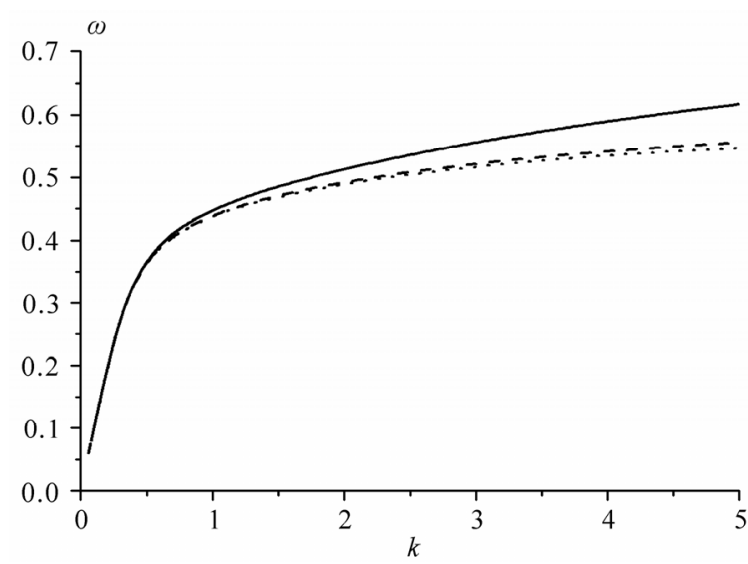

(a)

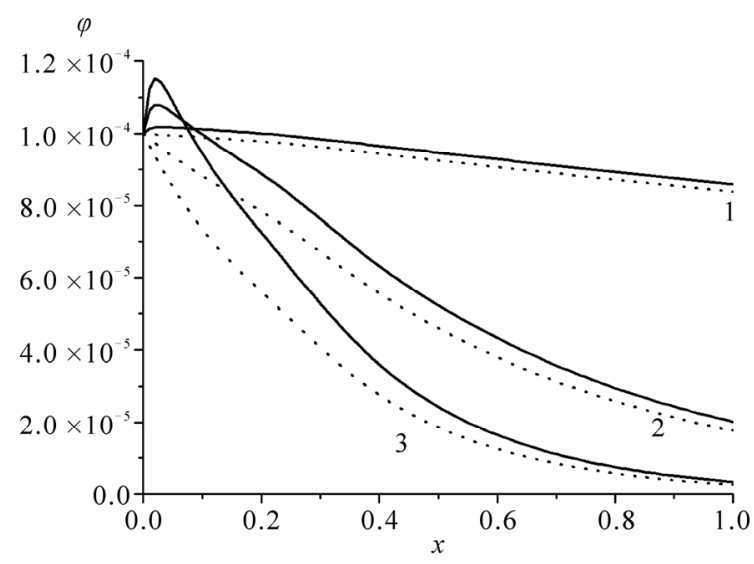

(a)

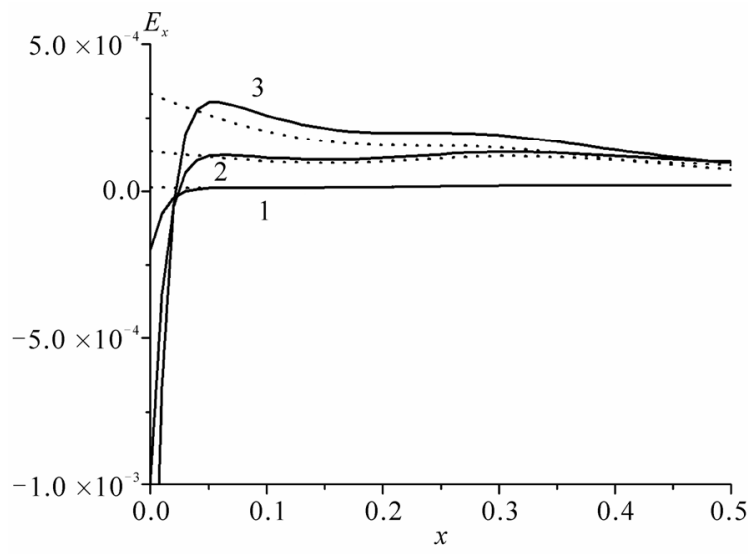

(c)

Figure 5. The dispersion curve $\omega(k)(a)$, transverse profiles of the potential $\varphi(b), x$-component of the electric field $E_{x}$ (c). Solid curves are for $T=10^{-4}$, dash curves are for $T=$ $10^{-6}$, dot curves are for $T=0$. The parameters are as follows: $a_{0}=-0.5$ (lighter particles in the center of the waveguide), $x_{0}=0.25, \varepsilon=5, p=2$ (step-like profile of the mass). The curves $1,2,3$ correspond to the values of the wave numbers $k=0.4, k=2, k=4$. realized for different dust temperatures $T=10^{-3} \cdots 10^{-6}$, and also for $T=0$.

For uniform semi-infinite dusty plasma, the results of simulations coincide with well-known data on surface dust acoustic waves, see Figure 2. It is seen that the surface plasma waves are preferably oscillations of the surface charge. The localization thickness of the charge near the surface is of about $x \sim 0.01 \cdots 0.02$.

But the simulations of the dusty plasmas with variable masses of the dust particles yield non-trivial results. One can see that when the dust particles are heavier in the center of the waveguide, it is possible to observe the negative group velocity of surface waves at some intervals of the wave numbers, see Figures 3, 4.

This result is tolerant to changes of parameters of the dusty plasma waveguide, both for Gaussian and step-like profiles of the masses of dust particles and also for different values of the dielectric permittivity of contacting dielectrics. When the finite temperature of the dust is taken into account, the negative dust velocity occurs at $T \leq$ $10^{-4}$, whereas at higher temperatures this phenomenon vanishes.

A possibility of opposite directions of phase and group velocities of the surface plasma waves is important for realization of wave instabilities, for instance, due to rotation of dust particles [2]. Namely, as known from the backward wave tube theory, the negative group velocity can lead to positive distributed feedback within the system $[14,15]$. The region of the wave numbers where the group velocity of the wave is close to zero and changes its sign can be also useful for observing nonlinear wave phenomena, especially, envelope solitons or modulation instability $[14,16]$.

In the case of lighter particles in the center of the waveguide, the group velocity is positive. But at some values of the wave numbers, the distribution of the transverse $E_{x}$ component of the electric field possesses non-monotonic dependence on the transverse coordinate $x$, see Figure 5.

This can be important for the dynamics of nonlinear waves in this waveguide. The results on negative group velocity have been confirmed by our more exact numerical simulations of hydrodynamic equations, where both the finite temperature of chaotic motion of dust particles and the finite mass of ions were taken into account.

\section{Conclusion}

To estimate the typical scales of the distance and time in simulation, it is necessary to use the following parameters: the temperature of the ions is $T_{i}=2000 \mathrm{~K}$, the ion concentration is $n_{i 0}=10^{3} \mathrm{~cm}^{-3}$, the radius of the dust particle is $r_{d}=10 \mu \mathrm{m}$, the mass of the dust particle far 
from the boundary is $m_{\infty}=2 \times 10^{-8} \mathrm{~g}$, the relation between the charges of the dust particle and the ion is $Q / e=10^{3}$. In this case, the Debye radius for the ions (and the characteristic spatial scale) is $r_{D i}=\left(k_{B} T_{i} / 4 \pi e^{2} n_{i 0}\right)^{1 / 2} \approx 10 \mathrm{~cm}$; the characteristic velocity is $V_{n}=\left(k_{B} T_{i} / m_{\infty} \times Q / e\right)^{1 / 2} \approx 0.1$ $\mathrm{cm} / \mathrm{s}$; the characteristic time is $t_{n}=r_{D} / V_{n} \approx 100 \mathrm{~s} \approx 1.6$ $\min$.

When the dust particles are heavier in the center of the waveguide, it is possible to observe the negative group velocity of surface waves. So, this case is a good condition for obtain of the absolute instability of dust surface plasma waves. This instability should be analyzed additionally. The estimated temporal and spatial scales have demonstrated that it could be possible an appearance of investigated phenomena under natural hazards, like volcano eruptions.

\section{Acknowledgements}

Authors are grateful to CONACyT (Mexico) for a partial financial support of our work.

\section{References}

[1] V., Fortov, A. Ivlev, S. Khrapak, A. Khrapak and G. Morfill G.E., "Complex (dusty) plasmas: Current status, open issues, perspectives", Physics Reports, Vol. 421, No 1-2, 2005, PP. 1-103.

[2] Shukla, P., Mamun, A. (2002) Introduction to Dusty Plasma Physics. Bristol, Institute of Physics Publ.

[3] Verheest F., (2000) Waves in Dusty Space Plasmas. Dordrecht, Kluwer Publ.

[4] P. Shukla and A. Mamun, "Solitons, shocks and vortices in dusty plasmas", New Journal of Physics, Vol. 5, No 1, 2003, PP.17.1-17.37.
[5] S. Popel, S. Kopnin, I. Kosarev, M. Yu, "Solitons in Earth's dusty mesosphere", Advances in Space Research, Vol. 37, No 2, 2006, PP. 414-419.

[6] P. Shukla, "A survey of dusty plasma physics", Physics of Plasmas, Vol. 8, No 5, 2001, PP. 1791-1803.

[7] K. Ostriko, M. Yu and L. Stenflo, "Surface waves in strongly irradiated dusty plasmas", Physical Review E, Vol. 61, No 1, 2000, PP. 782-787.

[8] B. Klumov, G. Morfill, S. Popel, "Formation of Structures in a Dusty Ionosphere", Journal of Experimental and Theoretical Physics, Vol. 100, No 1, 2005, PP. 152164.

[9] Kopnin S., Popel S. (2005) Dust Acoustic Mode Manifestations in Earth's Dusty Ionosphere, AIP Conference Proceedings, Vol. 799, PP. 161-164.

[10] S. Popel, M. Yu, V. Tsytovich, "Shock waves in plasmas containing variable charge impurities", Physics of Plasmas, Vol. 3, No 12, 1996, PP. 4313-4315.

[11] S. Popel, A. Golub', T. Losseva, R. Bingham and S. Benkadda, "Evolution of perturbation in charge-varying dusty plasmas", Physics of Plasmas, Vol. 8, No 5, 2001, PP. 1497-1504.

[12] V, Grimalsk, S. Koshevaya, R. Perez-Enriquez, A. Kotsarenko, "Interaction of linear and nonlinear ion-sound waves with inclusions of dusty plasma", Physica Scripta, Vol. 74, No 3, 2006, PP. 317-324.

[13] Samarskii, A., (2001) The Theory of Difference Schemes. N.Y., Marcel Dekker.

[14] Infeld, E., and Rowlands G. (2000) Nonlinear Waves, Solitons and Chaos. Cambridge University Press, Cambridge.

[15] Lifshitz, E., Pitaevskii, L. (1981) Physical Kinetics. London, Pergamon.

[16] Ostrovsky, L., Potapov, A., (2002) Modulated Waves: Theory and Applications. N.Y., The Johns Hopkins University Press. 